\title{
The Research of 3-D Modeling in the Virtual Campus
}

\author{
Xia Xiaotang \\ College of urban construction, Wuhan University of Science and Technology, Huangjia Lake Road, \\ Wuhan 430000, China \\ xiaxiaotang2010@qq.com
}

Keywords: Virtual Campus; 3D modeling; ARCGIS

Abstract. This paper indicates the process of 3D modeling in the building of virtual campus, which focus on the construction of terrain and scene modeling. Then it puts forward the cautions of these modeling processes. Finally, the 3D interaction and spatial analysis are involved.

\section{CONSTRUCTION OF THE TERRAIN MODEL}

With the spatial information and virtual reality technology development, the 3D virtual campus roaming has become a hotspot and frontier. Virtual roaming breaks the limits of the traditional planar map. Through digital terrain and object modeling, it provides the users an experience of virtual campus environment and 3D information service. University campus as a center of culture and sciences is the collection of university image window, which needs more science and technology as the guide. Therefore, introducing virtual roaming technology is very necessary. Three-dimension (3D) modeling is introduced to Geographic Information System (GIS). A strategy of the construction of 3D model, 3D visualization and 3D spatial analysis are proposed in Supernatural GIS environment ${ }^{[1]}$. Based on air photos and large scale topographical map, with Digital Photogrammetric System (DPS) and 3DMAX techniques, a 3D virtual scene of Henan Polytechnic University campus is designed and a virtual campus roaming system comes true, which provides users with all-round roaming in different perspective, spatial enquiries and analysis by intelligent man-machine interaction.

3D information of campus includes Digital Elevation Model (DEM), Digital Line Graph (DLG), Digital Orthophoto Map (DOM), the geometric feature points, texture data and image. DEM is a digital expression that reflects the surface of the terrain. DLG provides a basis for the spatial position of the terrain. The acquisition of the $3 \mathrm{D}$ geometric data is from the digital photogrammetric system, which help you maximally save money and improve work efficiency. Specific process is as follows: Firstly, getting the stereo image data by aerial photography. Secondly, do relative and absolute orientation process. Finally, high-precision DEM data is acquired by sampling of spatial data in the certain spacing and DLG is sketched. DOM can be acquired by using the DEM to correct aviation pictures or remote sensing image (monochrome/color). Because DOM contains landform, vegetation, roads, water system and the surface texture, the texture information of the 3D model can be extracted by DOM. The texture data is from extracting of features of texture in large batch processing according to the similar texture of objects in photography picture. By this means, the subsequent processing workload of texture is greatly reduced. Compared to the pure geometry data, vast storage space is occupied by photo texture. Therefore, how to decrease the storage space of image is a problem that must be resolved. Considering the symmetry and regularity of building 
appearance, texture segmentation is a good method. For example, the façade texture of windows, balcony and building are split up into the bitmap to store in the form of map symbol, which can be invoked at any time.

Virtual modeling is an important process. In order to facilitate modeling, the model is classified into terrain model, building model and complicated terrain model, according to the characteristics of topographical objects. Different model is separately constructed by corresponding method after unifying modeling scale ${ }^{[2]}$. This paper adopts digital photogrammetric technology, based on CAD and 3dmax development environment, constructs 3D model of campus landscape. According to the characteristics of building shape, the buildings are classified and coded uniformly. For every shape, corresponding geometric data model is adopted. For the complex building, such as domes, pointed roof building, although its overall appearance is different, it can be decomposed into two or more basic geometries, which are combined to express a complex of buildings.

Virtual campus is a concrete manifestation of digital Earth in campus region. At present, the GIS technology has been massively used in digital campus, and many universities have set up their own campus GIS systems, but these systems are mostly based on two-dimensional, although its function is perfect, but the terrain feature is not expressed visually and vividly. In recent years, three-dimensional GIS technology becomes fast mature and more and more been widely used in digital campus construction. Three-dimensional virtual campus has the lifelike visual effect, and it can vividly reveal the real campus landscape, realize the three dimensional terrain and the three dimensional landscape organic superposition, greatly increase the authenticity, and thus bring the users a strong visual impact for an immersive experience. The design method of three-dimensional virtual campus mainly has the following several kinds:

1) Take CAD, 3DSMAX as the platform to build three-dimensional model. In this way, the modeling effect is good, and it is suitable for small-scale models of small quantities of building landscape's construction, but it is imperfect to the terrain expression, generally it is only used in drawing up the three dimensional effect charts.

2) Use the ArcGIS, SuperMap and other software's three-dimensional functions, and carry on secondary development in the ArcGIS, SuperMap environment. This method has the advantage of no need using external modeling software, the shortcoming of this method is that it has high requirements on the programming, the modeling code is long, and the developed system cannot operate independently from the development environment. The main work of virtual campus system can be summarized as the following two aspects:

- Modeling three-dimensional campus scene. That is in the computer, according to the actual size, set up the three-dimensional model of the main features in campus, which form a virtual environment, including buildings, roads, trees, street lighting, planning green, artificial lakes, etc. 


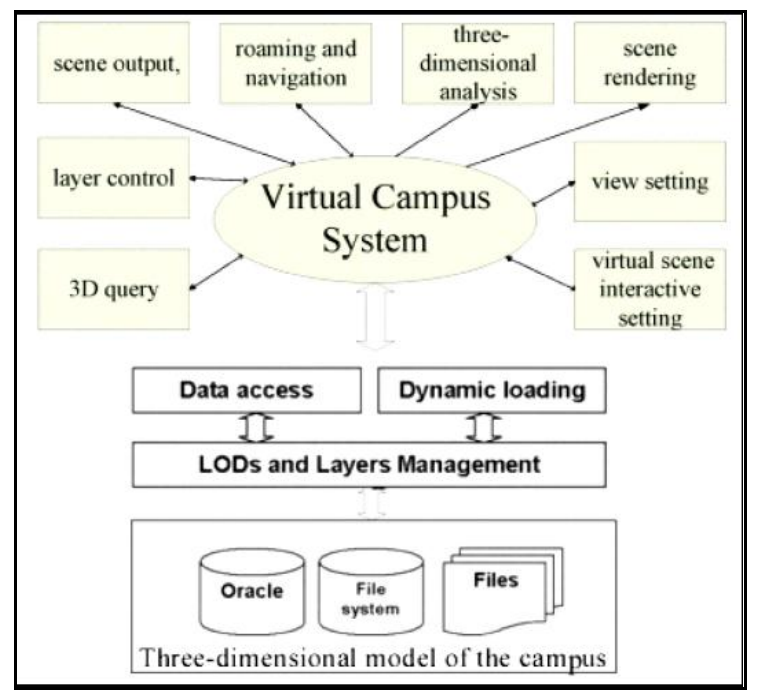

Figure 1. The general framework chart of virtual campus system

- The design and realization of the system. That is, to complete the overall design of the virtual campus system, and realize it in VC and OpenGL development environment by programming. The major function of the system includes roaming and navigation, view setting, virtual scene interactive setting, scene rendering, 3D query, three-dimensional analysis, scene output, layer control, etc.

\section{THE THREE-DIMENSIONAL CAMPUS SCENE MODELING BASED ON SKETCHUP}

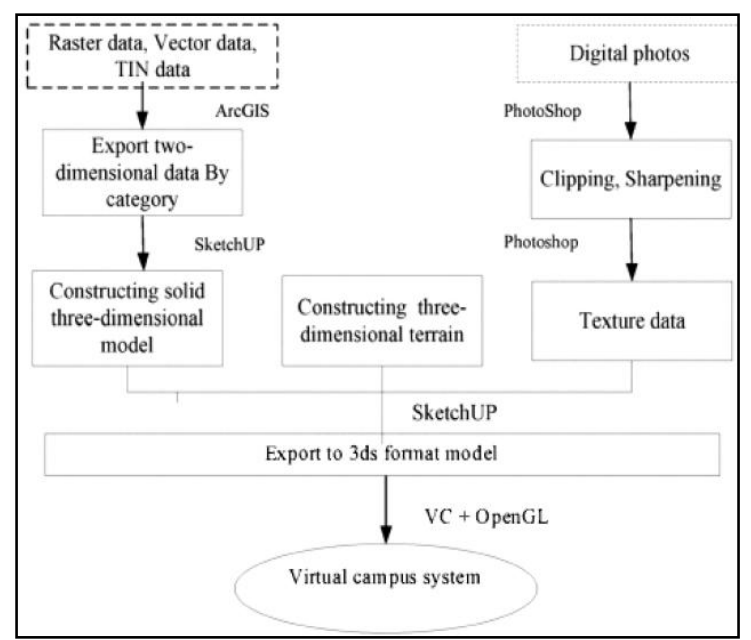

Figure 2. The flow chart of SketchUP three-dimensional modeling

Modeling Three-dimensional Campus Scene

- Two-dimensional data source

The study area is Wuhan University of Science and Technology. The two-dimensional data source includes campus two-dimensional ichnography, campus remote sensing topographic map, 
building's elevation data and real photos. Among them, the campus two-dimensional ichnography is from the campus planning CAD data, and processed it into ArcGIS 10.0 in Shape format data. The campus remote sensing topographic map is collected from satellite images on Google Earth software ${ }^{[3]}$. Use Universal Trans verse Mercator projection WGS84 coordinate system. Correcting and registration the campus shape files and campus remote sensing topographic map through using eight control points which are selected within the campus.

\section{- Three-dimensional modeling in SketchUp}

Three-dimensional modeling of plants, lights, signal board and other point features Campus plants, lights, signal board and so on are point features, which are can only be seen as point position but cannot been identified what is the each point represents. Therefore, it requires adding the correct models according to the original CAD annotation and labels symbols data, and then reviews it with the actual campus situation. If there is any difference, then modify the models to ensure the established three-dimensional campus model is consistent with the actual situation as far as possible. In the modeling process, we should have the objects, which have same attributes into one group in time, which would help to save as a record for the same and will be helpful for the analysis and the management in the virtual campus system.

Three-dimensional modeling of roads, rivers and other linear feature Campus roads, river and so on are linear feature, and these lines are three-dimensional surface with a certain area needs to be extended based on the actual width. For the road in the rolling terrain modeling, using the Drape tool in Sandbox tool bar, then may realize the projection form the flat path to the rough path. Rivers are different from roads; generally, they are lower than the surrounding terrain. At this time, it needs to be reversed stretching along the Z-axis.

Cautions in Modeling Process

Virtual scene modeling is the basis of the virtual campus system. The model directly influences the efficiency of the system running and the fidelity of the scene ${ }^{[4]}$. Use SketchUp to model the geographical features should pay attention to the following questions?

- Select the correct coordinate system. The created three-dimensional model should be consistent with GIS coordinates?

- When large-scale three-dimensional scene is created, the model data cannot be too large; Otherwise, it will affect the system running speed. In the modeling process, it often need get balance between the detail and complexity of the model, as much as possible to simplify the model, reducing the number of edges and faces; using texture images instead of models detail; The texture image's resolution should not be too high, and the maximum size of the texture image should not exceed $120 \mathrm{~KB}$ ?

- The utilization of instances. In complex scenes, since they will use massive same geometric solid (such as street lamps) and will make the geometric solid quantity increase rapidly, this will greatly increase the storage space. Using instances can solve this problem. Instances like numerous shadow of a model, the actual object is one, besides space location, the other property are same. Through the utilization of instances, it can not only save the modeling time but also reduce the amount of data. 


\section{D INTERACTION AND SPATIAL ANALYSIS}

Based on Supernatural GIS, the Campus landscape roaming can be set along a certain path to reach a target or choose the way objects move such as fixed path, interactive operation and so on. The 3D scene of Campus can be observed instantly from different point of view.3D roaming can make users have an immersive feel like sitting in the scenes and simulating driving or flight. The attributes of objects also can be inquired. Choosing the graphic ID, a database of the entity is obtained, and then the corresponding attribute of every object can be inquired, such as the name, height, usage, layer, building structure and coordinate. In addition, the function of powerful edit is provided by the roaming system. Graphical edit includes newly building, changing, deleting, changing the objects. Hitting the selected ID of entity, the database of attribute will be opened to lookup and locate the record to operate. If the location fails, an error message will be shown, or else delete the record.

The integration of GIS with 3D modeling technology easily realizes 3D information query, 3D spatial analysis, 3D dynamic interaction and spatial management of campus.3D virtual campus roaming expands the time and space of the campus, makes education process overall information-based and raises the teaching quantity, research and management level.

\section{References}

[1] T.Burger,W.Busch. Using Knowledge about Shape and Position of Plant Elements in Photogrammetric As-Built-Documentation. The 19th Congress of the International Society for Photogrammetry and Remote Sension (ISPRS), Amsterdam,2000, vol.33,pp.107-113.

[2] R.Shibasaki, A.Takuma,Z. Huijing.A mobile user interface for 3D spatial database based on the fusion of live landscape imagety. Proceedings of UM3'98 International Workshop on Urban Malti-Media/3D Mapping, Tokyo,1999,pp.23-30.

[3] Haist.J,Ramos.H, M.Figueiredo, Reitz, T.Symbology Encoding for 3D GIS-An approach to extend 3D Campus model visualization to GIS visualization. Proceedings of the Urban and Regional Data Management. UDMS Annual,2008:P121-131

[4] LIU Hang, WANG Ji-zhong and WANG Chun-shui," Key technology study on 3D simulation system of virtual campus," Computer Engineering and Design, voI.28,pp.2934-2936,June 2007. 